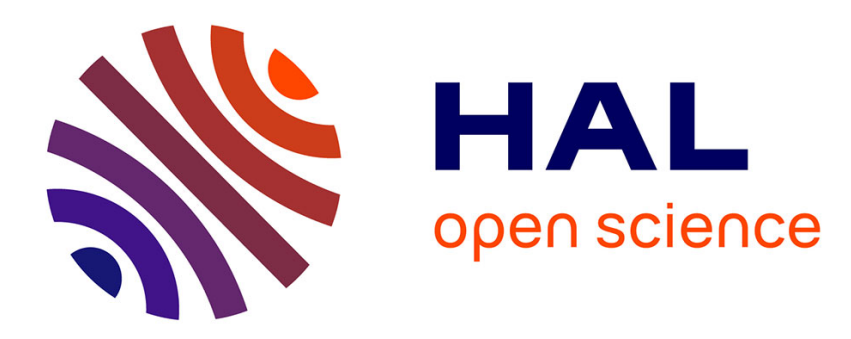

\title{
Origine microstructurale de l'amorçage des fissures intergranulaires dans les alliages Al-Li 8090
}

\author{
Catherine Verdu, R. Fougères
}

\section{To cite this version:}

Catherine Verdu, R. Fougères. Origine microstructurale de l'amorçage des fissures intergranulaires dans les alliages Al-Li 8090. Revue de Physique Appliquée, 1990, 25 (11), pp.1095-1105. 10.1051/rphysap:0199000250110109500 . jpa-00246278

\section{HAL Id: jpa-00246278 https://hal.science/jpa-00246278}

Submitted on 1 Jan 1990

HAL is a multi-disciplinary open access archive for the deposit and dissemination of scientific research documents, whether they are published or not. The documents may come from teaching and research institutions in France or abroad, or from public or private research centers.
L'archive ouverte pluridisciplinaire HAL, est destinée au dépôt et à la diffusion de documents scientifiques de niveau recherche, publiés ou non, émanant des établissements d'enseignement et de recherche français ou étrangers, des laboratoires publics ou privés. 


\title{
Origine microstructurale de l'amorçage des fissures intergranulaires dans les alliages Al-Li 8090
}

\author{
C. Verdu et R. Fougères \\ GEMPPM, UA CNRS n 341, INSA Lyon, 69621 Villeurbanne Cedex, France
}

(Reçu le 21 mars 1990, révisé le 5 juillet 1990, accepté le 13 juillet 1990)

\begin{abstract}
Résumé. - L'objectif de ce travail était de comprendre et de modéliser l'amorçage des fissures dans les alliages $\mathrm{Al}-\mathrm{Li} 8090$ sollicités en traction monotone. Une attention particulière a été portée sur les fissures intergranulaires - ou de délaminage - . Nous avons montré que la forte localisation de la déformation sur un seul système de glissement, ainsi que l'apparition d'un glissement " sinueux » et intense résultant probablement des incompatibilités de déformation de grain à grain, sont à l'origine de l'amorçage des fissures, et cela quelles que soient les conditions de revenu. Nous avons montré aussi que la précipitation intergranulaire augmente le risque de délaminage. Mais l'interaction glissement-joints de grains semble tout de même être responsable de l'amorçage intergranulaire. Enfin, nous avons développé pour les états sous revenus une analyse quantitative basée sur un modèle d'empilements de dislocations contre les joints de grains qui est en accord avec les résultats expérimentaux.
\end{abstract}

\begin{abstract}
This study is concerned with crack origin of $8090 \mathrm{Al}-\mathrm{Li}$ alloys tensile tested. Particular attention has been given on grain boundary crack or delamination. It has been shown that the origin of the fracture is due to a strong slip localisation on a one single slip system and to the appearance of a sinuous and coarse slip probably resulting from incompatible strains between adjacent grains, whatever the ageing conditions. Also, it has been shown that the intergranular precipitation increases the risk of intergranular cracks. But the slip localisation seems to be responsible for the delamination initiation. In the case of underaged condition a quantitative analysis from a model of dislocations piled up against grain boundary has been developed. This approach agrees with experimental results.
\end{abstract}

\section{Introduction.}

Il est bien connu que, dans les alliages $\mathrm{Al}-\mathrm{Li}$ présentant des grains allongés par filage ou laminage, des fissures intergranulaires se produisent fréquemment selon une direction parallèle à l'axe de traction [1-5]. Ce phénomène est appelé délaminage.

Ce mode de rupture particulier a été attribué à 1 erents acteurs, comme a oca isa ion e a déformation dans des bandes de déformation [6], la présence de zones dénudées de précipités au voisinage des joints de grains [7], les ségrégations d'éléments résiduels, sodium ou potassium dans les joints de grains [8] ou à l'effet de la ségrégation du lithium lui-même [9]. D'autres auteurs [10] ont prétendu que la localisation de la déformation et la précipitation intergranulaire jouent un rôle important respectivement dans les états sous-revenus et sur-revenus. Une étude récente [11], concernant le délaminage se produisant lors d'essais de flexion, indique que les causes de fragilité intrinsèques au joint de grain lui-même ne peuvent être associées aux ruptures de délaminage et que la localisation de la déformation semble jouer un rôle majeur.

Le but de cet article est d'étudier l'effet des modes de déformation plastique sur les ruptures inter et intragranulaires dans les alliages Al-Li 8090 pour

\section{Procédure expérimentale.}

Les éprouvettes ont été prélevées dans un larget filé de $13 \mathrm{~mm}$ d'épaisseur, soit dans le sens de la direction de filage, sens long (L), soit dans le sens perpendiculaire à la direction de filage, sens travers long (TL) (Fig. 1).

Comme après traitement thermique la surface et les bords du larget peuvent présenter une recristallisation partielle $[12,13]$, les éprouvettes ont été usinées au cœur de celui-ci. La partie utile des 


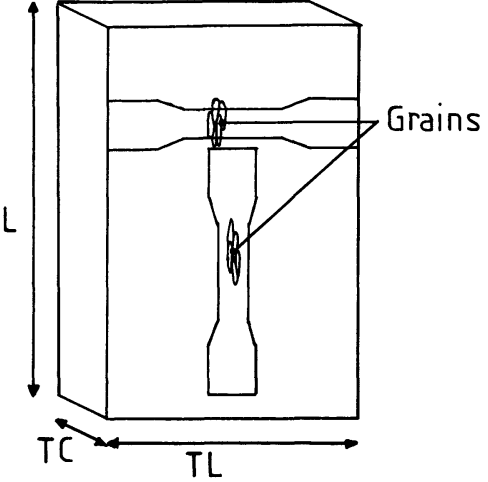

Fig. 1. - Sens de prélèvement des éprouvettes.

[Taking directions of the samples.]

éprouvettes est un parallélépipède de $30 \mathrm{~mm}$ de longueur, de $6 \mathrm{~mm}$ de largeur et de $6 \mathrm{~mm}$ d'épaisseur.

Avant usinage, nous effectuons un traitement de mise en solution de $2 \mathrm{~h}$ à $540{ }^{\circ} \mathrm{C}$ en bain de sel, puis une trempe à l'eau et enfin nous procédons soit à une maturation à température ambiante supérieure à un mois, soit à un revenu à $190^{\circ} \mathrm{C}$ d'une durée variable afin d'obtenir des états : sous-revenus $(6 \mathrm{~h}$ à $\left.190^{\circ} \mathrm{C}\right)$, revenus au pic de durcissement $(12 \mathrm{~h}$ à $\left.190{ }^{\circ} \mathrm{C}\right)$ et sur-revenus $\left(31 \mathrm{~h}, 48 \mathrm{~h}\right.$ et $96 \mathrm{~h}$ à $\left.190^{\circ} \mathrm{C}\right)$.

En raison de la présence dans l'alliage de zirconium qui inhibe la recristallisation, l'alliage présente une structure de grains allongés dans la direction de filage; longueur de 1 à $8 \mathrm{~mm}$, largeur de 250 à $500 \mu \mathrm{m}$, épaisseur de 20 à $100 \mu \mathrm{m}$ ainsi que des sous grains de polygonisation de taille moyenne de 5 à $10 \mu \mathrm{m}$ (les sous grains sont cependant légèrement allongés dans la direction de filage).

Les observations effectuées au MET nous ont permis de retrouver les caractéristiques microstructurales de cet alliage, définies dans la littérature $[3,10$, 14]. Quel que soit le traitement thermique (maturation à température ambiante ou revenu à $190^{\circ} \mathrm{C}$ ), les échantillons présentent tous une précipitation homogène $\delta^{\prime}\left(\mathrm{Al}_{3}-\mathrm{Li}\right)$. Pour l'état sous-revenu, la précipitation $\mathrm{S}^{\prime}\left(\mathrm{Al}_{2} \mathrm{CuMg}\right)$ apparaît sur les dislocations et les sous-joints de grains. Les précipités $S^{\prime}$ deviennent abondants pour les états sur-revenus. Pour ce même état, une importante précipitation $\mathrm{T}_{2}\left(\mathrm{Al}_{6} \mathrm{Cu}(\mathrm{Li}, \mathrm{Mg})_{3}\right)$ est observée aux joints de grain.

Les éprouvettes ont été sollicitées en traction monotone à température ambiante, sur une machine MTS de type 810 , avec une vitesse de déformation de $3 \times 10^{-4} \cdot \mathrm{s}^{-1}$.

Afin de mettre en évidence la relation entre modes de déformation plastique et modes de rupture, nous avons adopté la méthodologie expérimentale suivante:

- à l'aide d'une binoculaire montée sur la machine de traction, nous avons observé la surface polie de l'éprouvette (plan long-travers long (L-TL)) au cours de l'essai, afin d'étudier les bandes de glissement pendant la déformation plastique sous un grossissement de $\mathrm{X} 80$;

- puis, les faciès de rupture sont analysés à l'aide d'un microscope électronique à balayage (MEB Jeol $840 \mathrm{~A}$ ). Nous cherchons d'abord à mettre en évidence la relation entre les bandes de glissement et les caractéristiques des surfaces de rupture, au voisinage de la surface polie de l'échantillon. Enfin, nous vérifions qu'au cœur de l'échantillon les surfaces de rupture ont bien les mêmes caractéristiques que celles observées en surface afin de s'assurer que la corrélation établie entre plasticité et rupture est valable dans tout le volume de l'échantillon;

- enfin, des observations au microscope électronique à transmission (MET), JEOL $200 \mathrm{CX}$, sont réalisées sur des lames prélevées près de la surface de rupture, afin d'analyser, au niveau microscopique, l'effet de la déformation plastique et les configurations microscopiques critiques qui conduisent à la rupture.

Les résultats présentés ci-dessous sont issus des observations effectuées sur au moins trois éprouvettes pour chaque traitement thermique.

\section{Résultats.}

3.1 RÉSEAUX DE BANDES DE GLISSEMENT A LA SURFACE DES ÉCHANTILLONS. - Quel que soit le sens de prélèvement des éprouvettes, sens long ou travers long, les bandes de glissement observées à la surface (plan $L \times T L$ ) sont sensiblement les mêmes.

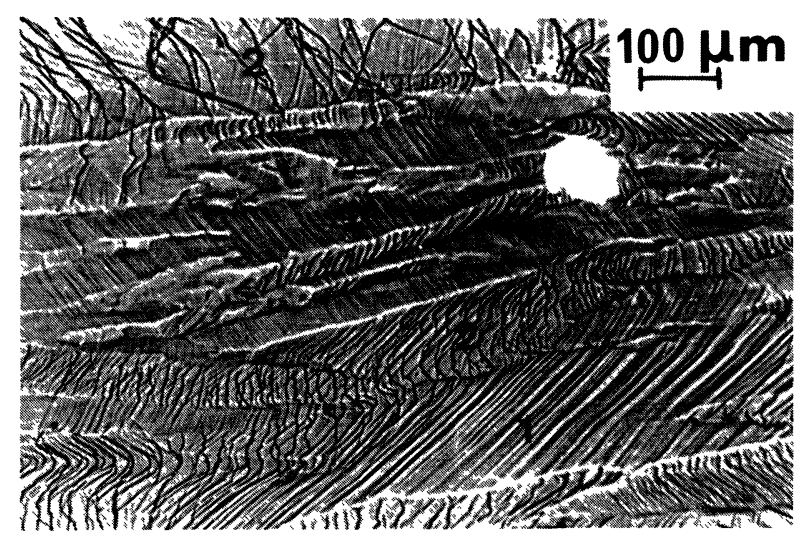

Fig. 2. - Micrographie optique du réseau de bandes de glissement à la surface polie des échantillons (revenu au pic, $12 \mathrm{~h}$ à $\left.190^{\circ} \mathrm{C}, \varepsilon_{\mathrm{p}}=0,08\right)$. Glissement rectiligne (1). Glissement sinueux (2).

[Optical micrograph of the slip line pattern at the prepolished surface (peak aged, $12 \mathrm{~h}$ at $190{ }^{\circ} \mathrm{C}, \varepsilon_{\mathrm{p}}=0.08$ ). Straight slip (1). Sinuous slip (2).] 
Deux types de glissement sont observés (Fig. 2) :

i) un premier système apparaît dans un grand nombre de grains sous forme de bandes de glissement rectilignes et réparties de manière homogène sur toute la surface libre du grain (voir Fig. 2 repère 1). L'angle que font ces bandes avec le joint de grain parallèle à la direction de filage varie de 20 à $60^{\circ}$ selon les joints. Bien que la majorité des grains ne présentent qu'un seul système de glissement, il faut noter que, dans quelques grains, deux ou trois systèmes sont activés alors que dans quelques autres aucune déformation plastique n'apparaît ; ii) lorsque la déformation plastique augmente, il apparaît un glissement qui, par observation optique, se présente sous une forme sinueuse (voir Fig. 2 repère 2). Par simplicité, ce type de glissement sera appelé glissement sinueux dans la suite de l'article.

Il est observé alors que le glissemènt rectiligne a été activé dans la majorité des grains et pour des déformations vingt fois supérieures à celles correspondant à l'apparition du glissement rectiligne (Tabl. I). On remarquera que dans les états surrevenus les lignes de glissement ne sont visibles que pour des déformations plastiques relativement importantes.

Tableau I. - Valeurs de la déformation plastique et de la contrainte pour lesquelles apparaissent au microscope optique les deux types de glissement. (Grossissement $=\times 80)$. Valeurs de la limite d'élasticité à $0,2 \%$.

[Strain and stress values for the emergence of the two kinds of slip observed with a light microscope (magnification $\times 80$ ). Yield strength values.]

\begin{tabular}{|c|c|c|c|c|c|}
\hline \multirow[t]{2}{*}{$\begin{array}{l}\text { Traitement } \\
\text { thermique } \\
\text { de revenu }\end{array}$} & \multicolumn{2}{|c|}{$\begin{array}{l}\text { Apparition } \\
\text { du glissement } \\
\text { rectiligne }\end{array}$} & \multicolumn{2}{|c|}{$\begin{array}{c}\text { Apparition } \\
\text { du glissement } \\
\text { sinueux }\end{array}$} & \multirow[t]{2}{*}{$\begin{array}{c}\sigma_{0,2} \% \\
\text { en } \mathrm{MPa}\end{array}$} \\
\hline & $\varepsilon_{\mathrm{p}}$ en $\%$ & $\sigma$ en $\mathrm{MPa}$ & $\varepsilon_{\mathrm{p}}$ en $\%$ & $\sigma$ en $\mathrm{MPa}$ & \\
\hline Revenu $2 \mathrm{~h}$ à $190^{\circ} \mathrm{C}$ & 0,016 & 243 & 0,37 & 343 & 316 \\
\hline Revenu $6 \mathrm{~h}$ à $190^{\circ} \mathrm{C}$ & 0,016 & 300 & 0,43 & 397 & 371 \\
\hline Revenu 11 h 30 à $190^{\circ} \mathrm{C}$ & 0,022 & 343 & 0,33 & 421 & 403 \\
\hline Revenu $31 \mathrm{~h}$ à $190^{\circ} \mathrm{C}$ & 0,09 & 400 & 0,75 & 438 & 420 \\
\hline Revenu $168 \mathrm{~h}$ à $190^{\circ} \mathrm{C}$ & 0,08 & 400 & 1,6 (traces) & 438 & 414 \\
\hline
\end{tabular}

Le glissement sinueux est très intense, et contrairement aux lignes de glissement rectiligne qui apparaissent progressivement et s'intensifient lorsque la déformation augmente, les lignes de glissement sinueux apparaissent de manière soudaine.

A l'intérieur des grains présentant de tels glissements, leur répartition est hétérogène. Dans certains grains ces glissements ont tendance à se mettre parallèles aux joints de grains.

D'une manière générale tous les grains de la surface d'une éprouvette ne présentent pas de tels glissements.

Le nombre de glissements sinueux augmente avec la déformation plastique, on observe parfois deux familles de ce type de glissement. Cependant lors de la rupture de l'éprouvette une partie des grains seulement est affectée par des glissements sinueux. Leur proportion est très forte pour les états maturé et sous-revenu, elle devient plus faible pour l'état revenu au pic; quelquées glissements sinueux apparaissent avant la rupture d'un échantillon surrevenu.
3.2 ObSERVATIONS AU MET. - Comme cela a déjà été mentionné par d'autres auteurs [6-11], nous . avons mis en évidence une forte localisation de la déformation dans des bandes correspondant aux traces de plans de glissement de type $\{111\}$ (Fig. 3).

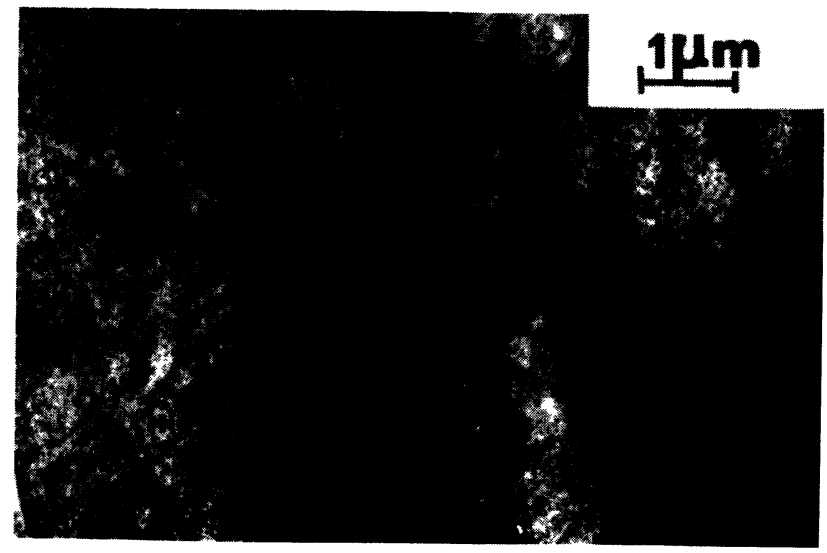

Fig. 3. - MET, échantillon sous-revenu $\left(6 \mathrm{~h}\right.$ à $\left.190^{\circ} \mathrm{C}\right)$, $\varepsilon_{\mathrm{p}}=0,09$. Bandes de déformation de type $\{111\}$.

[TEM micrograph of underaged specimen $\left(6 \mathrm{~h}\right.$ at $\left.190{ }^{\circ} \mathrm{C}\right)$, $\varepsilon_{\mathrm{p}}=0.09$. $\{111\}$ slip bands.] 
Dans l'état revenu au pic de durcissement, certaines de ces bandes sont très intenses (Fig. 4a). Dans le cas où le plan [111], dont la bande est la trace, n'est pas mis parallèle au faisceau d'électrons, le contraste obtenu est compatible avec un contraste de variation d'épaisseur (Fig. 4b), correspondant probablement à un cisaillement sur toute l'épaisseur de la lame. D'une manière générale, ces bandes débouchent soit dans le trou de la lame (Fig. $4 \mathrm{a}$, repère 1), soit sur une fissure intergranulaire (Fig. 4b), laissant des marches correspondant au passage d'environ 1500 dislocations (Figs. 4a et 4b). Aucune dislocation n'est visible dans ces bandes ; elles ont probablement été éliminées lors de la préparation de la lame. La présence de ces bandes et celle des marches qu'elles créent aux joints de grains restent tout de même la preuve d'une très forte localisation de la déformation et d'une accumulation contre les joints de grains d'un grand nombre de dislocations dans ces bandes. Enfin on observe un fort cisaillement des sous joints de grains par ces bandes (Fig. 4a, repère 2).

On observe aussi des bandes non rectilignes qui correspondent peut-être à l'image microscopique du glissement sinueux observé à la surface de l'échantillon (Fig. 4a, repère 3).

3.3 FACIÈS DE RUPTURE. - Sur la figure 5, nous avons schématisé la forme des éprouvettes rompues en fonction du temps de revenu. On a pu mettre en évidence que l'orientation de la surface de rupture avec l'axe de traction varie de $45^{\circ}$ pour l'état mûri à température ambiante, à $90^{\circ}$ pour les états revenus au pic et sur-revenus.

Cependant, quel que soit le traitement thermique, on observe des surfaces de ruptures intergranulaires
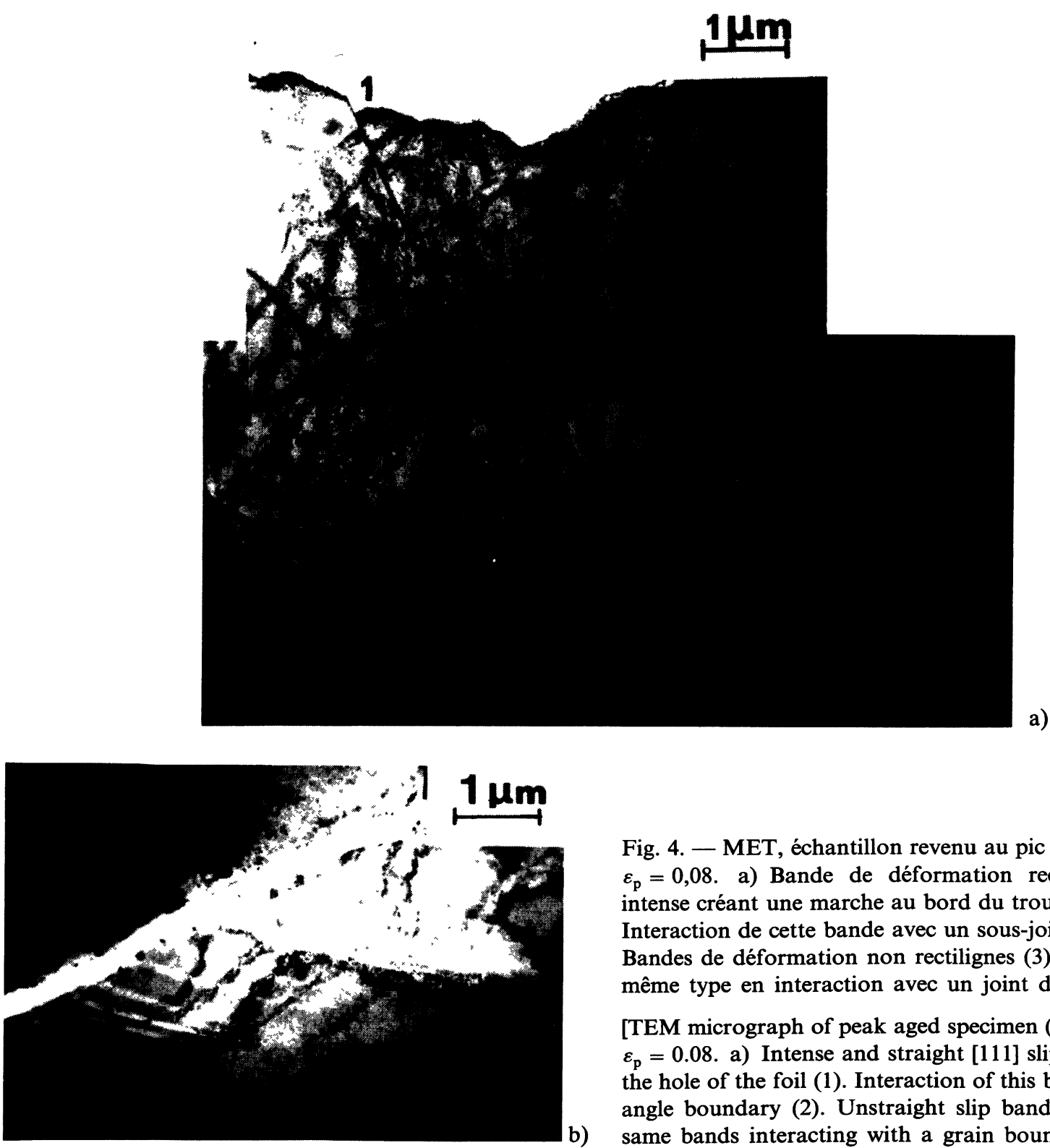

Fig. 4. - MET, échantillon revenu au pic $\left(12 \mathrm{~h}\right.$ à $\left.190^{\circ} \mathrm{C}\right)$, $\varepsilon_{\mathrm{p}}=0,08$. a) Bande de déformation rectiligne et très intense créant une marche au bord du trou de la lame (1). Interaction de cette bande avec un sous-joint de grain (2). Bandes de déformation non rectilignes (3). b) Bandes du même type en interaction avec un joint de grain.

[TEM micrograph of peak aged specimen $\left(12 \mathrm{~h}\right.$ at $\left.190{ }^{\circ} \mathrm{C}\right)$, $\varepsilon_{\mathrm{p}}=0.08$. a) Intense and straight [111] slip band. Step at the hole of the foil (1). Interaction of this band with a low angle boundary (2). Unstraight slip bands (3). b) Other same bands interacting with a grain boundary.] 


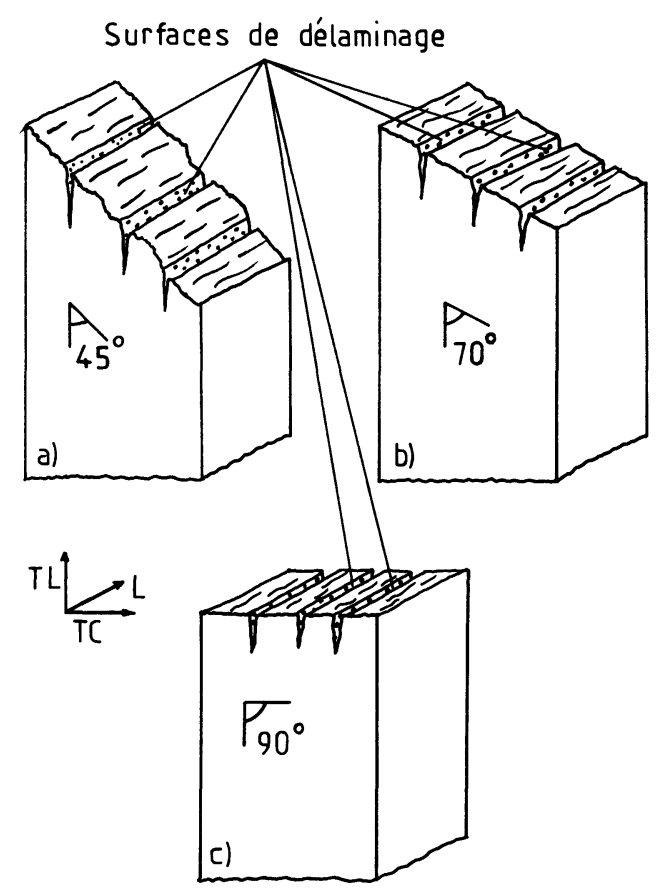

Fig. 5. - Schéma d'évolution de la forme des éprouvettes rompues avec le temps de revenu : a) maturé à température ambiante, b) revenu $6 \mathrm{~h}$ à $\left.190^{\circ} \mathrm{C}, \mathrm{c}\right)$ revenu $>$ à $6 \mathrm{~h}$ à $190{ }^{\circ} \mathrm{C}$. Direction de traction: TL.

[Schematical aspects of the broken tensile specimen with the ageing time : a) aged at room temperature, b) ageing $6 \mathrm{~h}$ at $\left.190^{\circ} \mathrm{C}, \mathrm{c}\right)$ ageing time $>6 \mathrm{~h}$ at $190{ }^{\circ} \mathrm{C}$. Tensile axis : TL.]

ou de délaminage, parallèles à l'axe de traction (Fig. 5).

3.3.1 Surfaces de délaminage (surfaces parallèles à la direction de traction). - La surface de délaminage ne présente pratiquement aucune cupule à l'état mûri. Par contre, celles-ci apparaissent avec le revenu du fait de la précipitation intergranulaire (Fig. 6b). Pour les états mûri et sous-revenu, de nombreuses traces de glissement et, notamment, de glissement sinueux sont présentes (Fig. 6a). Les marches provoquées par ces glissements sinueux sur la surface du joint de grain peuvent avoir une hauteur de $0,5 \mu \mathrm{m}$, ce qui correspond au passage d'environ 2000 dislocations. De plus, le relief o serve sur a sur ace, sor e e ace es presen an des contrastes différents, a été attribué aux sousjoints de grains, particulièrement nombreux dans ce type d'alliage non recristallisé. En effet, la taille des facettes correspond à celle des sous-grains. Dans le cas des états revenus au pic et sur-revenus, les traces de glissement sont moins marquées. Cependant, elles sont toujours présentes. En effet, sur la figure 7 , on peut voir la correspondance entre les lignes de glissement sur la surface polie de l'échantillon et le relief sur la surface de délaminage. Les traces de glissement sont probablement émoussées par la plasticité additionnelle dans la zone appauvrie en précipités $\delta^{\prime}$ au voisinage des précipités intergranulaires.

a)
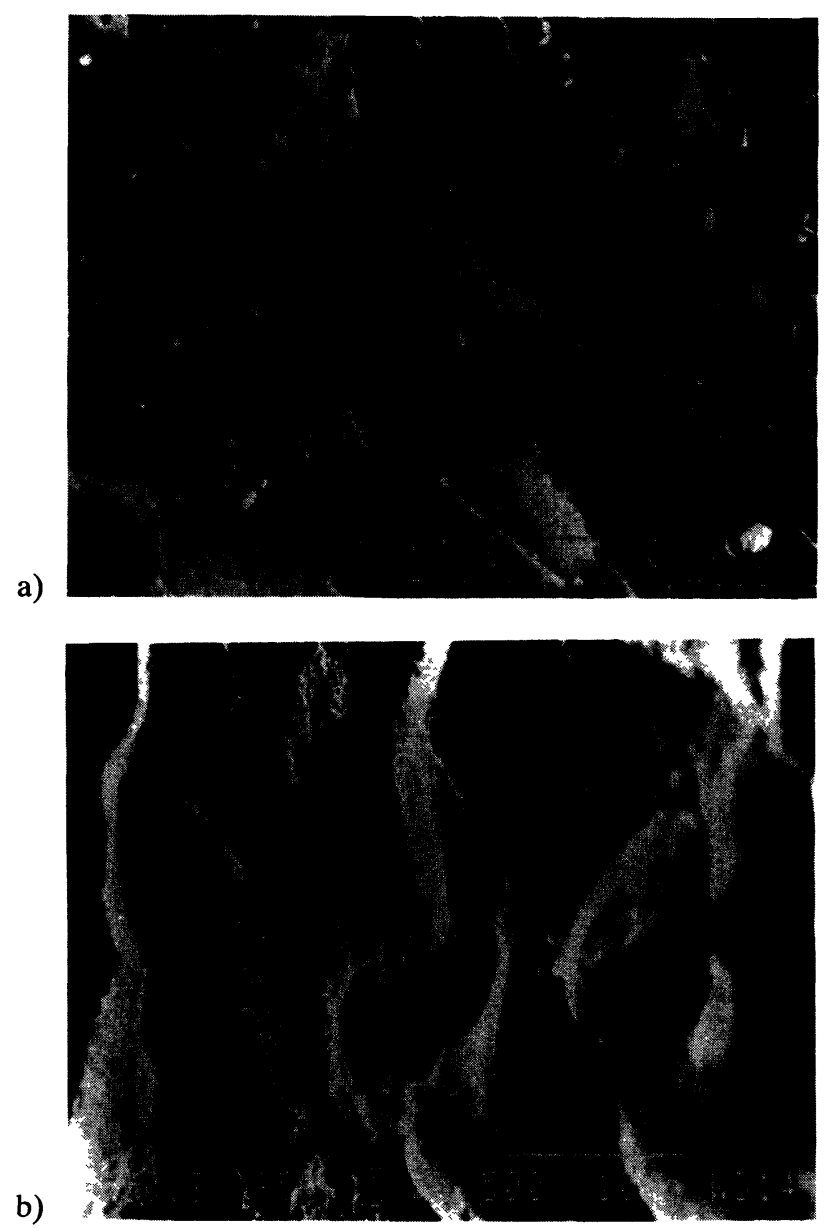

Fig. 6. - MEB, micrographie des surfaces de délaminage : a) sous-revenu; b) revenu au pic et sur revenu.

[SEM micrographs of grain boundary delamination surfaces : a) under aged, b) peak aged and over aged.]

3.3.2 Surface de rupture non parallèle à la direction de traction. - A partir des micrographies de la figure 8 , nous pouvons faire les remarques suivantes :

ans e cas e e a murı a empera ure am ian e, le mode de rupture est entièrement de type intragranulaire (Fig. 8a). Lorsque le temps de revenu augmente, des ruptures intergranulaires apparaissent et deviennent de plus en plus nombreuses. L'aspect de certaines zones semble même suggérer que la rupture se produit le long des sous-joints de grains (Fig. 8d). La figure 9 représente la proportion estimée de surface de rupture intragranulaire en fonction du temps de revenu à $190^{\circ} \mathrm{C}$. On peut voir qu'après 100 heures à $190{ }^{\circ} \mathrm{C}$, il n'y a plus que $50 \%$ de la surface qui est de type intragranulaire. Cette évolu- 


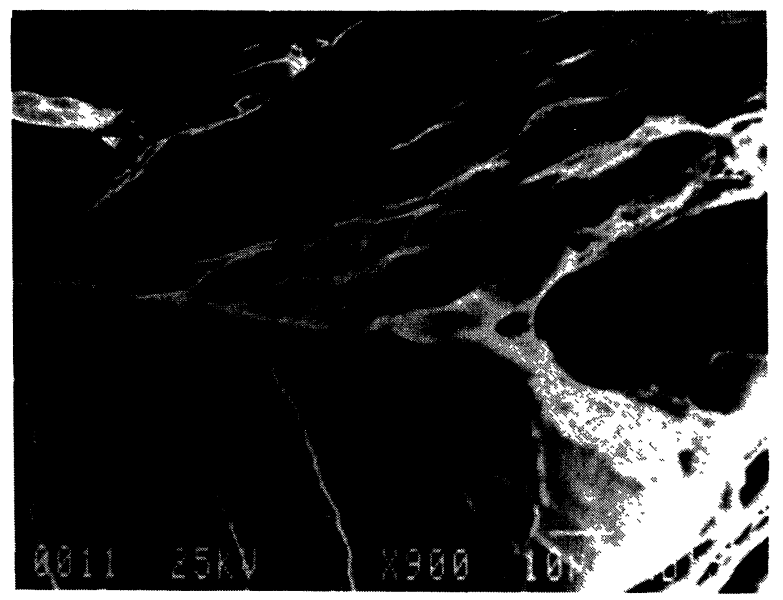

Fig. 7. - MEB : relation entre le relief de la surface de délaminage et le réseau de glissements sur la surface polie de l'échantillon. Cas d'un état sur-revenu ( $31 \mathrm{~h}$ à $\left.190^{\circ} \mathrm{C}\right)$.

[SEM : grain boundary delaminations and relation with slip line pattern at the prepolished surface. Case of an over aged specimen $\left(31 \mathrm{~h}\right.$ at $\left.190^{\circ} \mathrm{C}\right)$.]

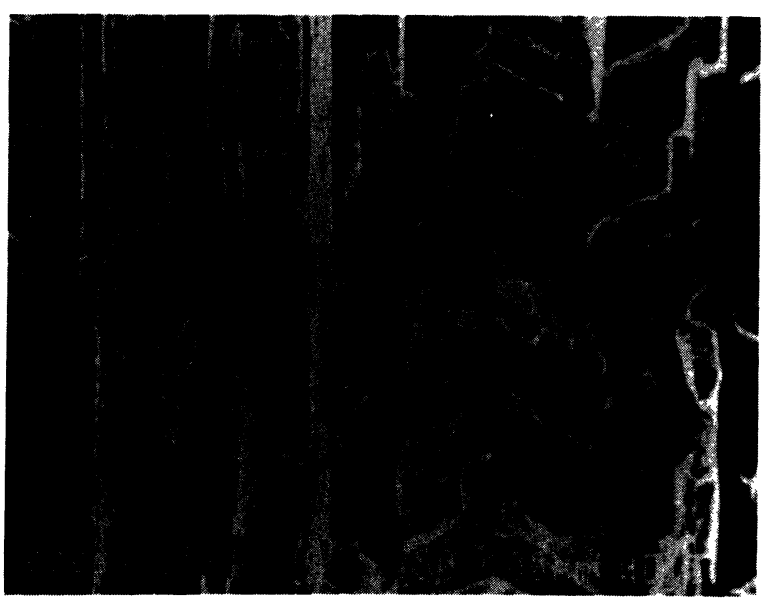

a)

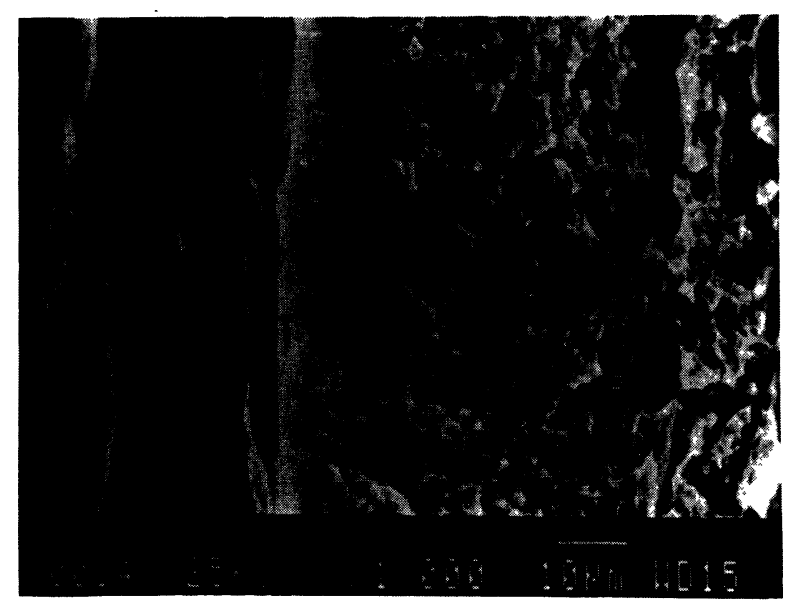

b) tion du mode de rupture avec le revenu a déjà été observé sur ce type d'alliage $[13,15]$ et sur l'alliage 2091 [16]. De plus, le fait que la proportion de ruptures intergranulaires augmente avec le temps de revenu, permet de comprendre l'évolution de l'orientation moyenne de la surface de rupture avec l'axe de traction. Ceci est probablement dû à une forte précipitation intergranulaire qui accroît le risque de rupture dans les joints. Dans les états maturé et sous-revenu, l'importance de la déformation plastique et l'orientation des lignes de glissement dans la structure à grains allongés favorisent la rupture intragranulaire et les ruptures intergranulaires pour les joints de grains parallèles à l'axe de traction. Pour l'état sur-revenu la précipitation intergranulaire est telle que même les joints de grains peu sollicités par la plasticité sont susceptibles de se rompre. Ce changement de mode d'amorçage de fissures explique probablement le changement dans l'orientation moyenne de la surface de rupture. En effet dans l'état sur revenu la taille de fissures intergranulaires

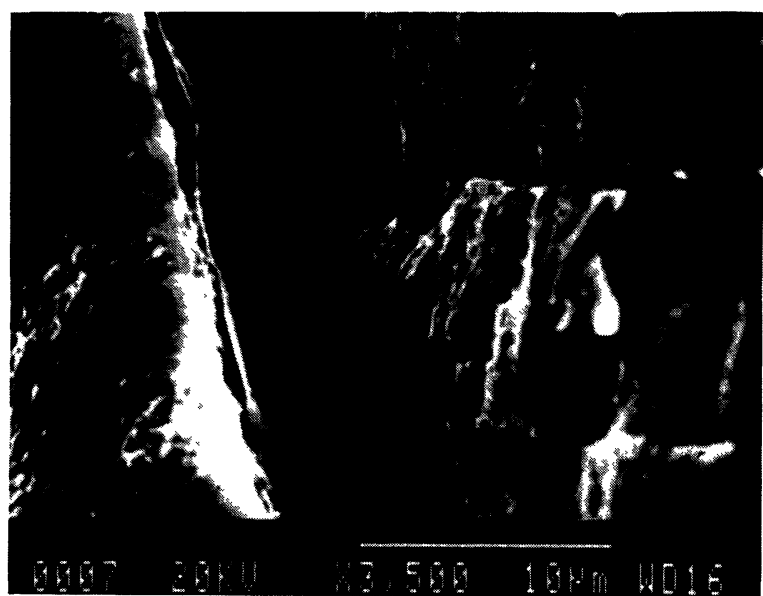

c)

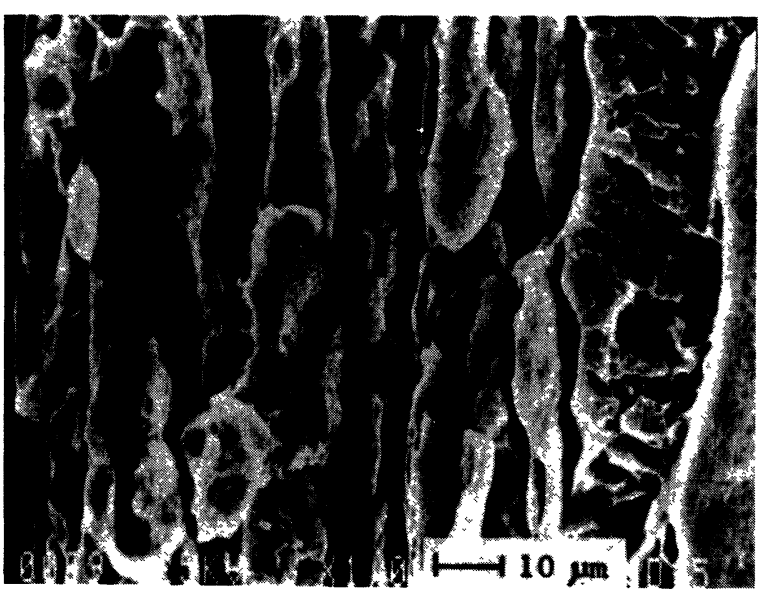

d)

Fig. 8. - MEB, surfaces de rupture non parallèles à l'axe de traction : a) rupture intragranulaire dans le cas d'état maturé à température ambiante, b) rupture intragranulaire dans le cas d'un sur-revenu, c) rupture intergranulaire à un point triple, d) rupture inter-subgranulaire (c et $d$ états sur revenus).

[MEB micrograph of fracture surfaces not parallel to the tensile axis: a) transgranular mode, aged at room temperature, b) transgranular mode, over aged condition, c) intergranular fracture at triple point, d) intersubgranular fracture (c and $\mathrm{d}$ : overaged specimens).] 


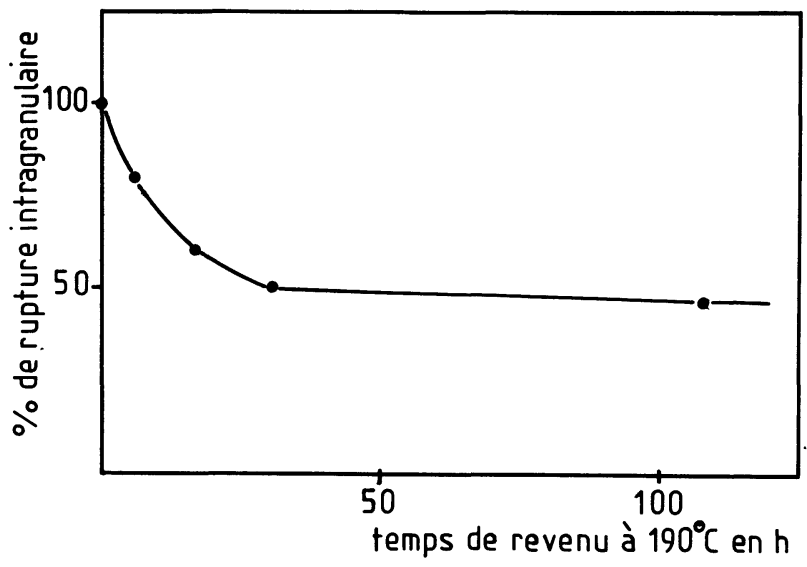

Fig. 9. - Proportion de surfaces de ruptures intragranulaires en fonction du temps de revenu à $190^{\circ} \mathrm{C}$.

[Pourcentage of transgranular fracture surfaces with the ageing time at $190{ }^{\circ} \mathrm{C}$.]

perpendiculaires à l'axe de traction est sans doute suffisante pour développer une fissure macroscopique dans cette direction.

$\mathrm{Si}$ on observe plus en détail les ruptures intergranulaires, on peut voir, au niveau des points triples, de grosses cavités bordées de zones sans cupules (Fig. 8c). Ces cavités sont probablement dues à la présence de gros précipités intergranulaires aux points triples. Cela vient confirmer l'explication du paragraphe précédent.

Quant à la rupture de type intragranulaire, son aspect évolue avec le temps de revenu. En effet, pour les états faiblement revenus, des arêtes rectilignes et parallèles sont visibles sur la surface de rupture. Nous avons pu corréler l'existence de ces arêtes avec le glissement sinueux visible sur la surface polie de l'échantillon ou sur les surfaces de délaminage au cœur de l'échantillon (Fig. 10). En

Fig. 10. - MEB : relation entre la rupture intragranulaire et le double réseau de glissement sinueux sur la surface de délaminage.

[SEM : relation between transgranular fracture and sinuous and coarse slip lines.] effet sur la figure 10 il apparait que la rupture se produit le long des glissements sinueux qui se développent selon deux systèmes différents. Entre ces arêtes, une sous-structure est visible et pour les temps de revenu plus importants, elle envahit la surface en lui donnant un aspect d'éponge (Fig. 8b).

\subsection{AMORÇAGE DES FISSURES INTERGRANULAIRES} - Nous avons observé la surface de l'éprouvette correspondant au plan travers-long, travers-court (selon l'épaisseur du larget (voir Fig. 1)), dans le but de déterminer l'origine des fissures intergranulaires.

Deux mécanismes d'amorçage semblent être présents. Dans le cas des états faiblement revenus, les fissures intergranulaịes sont droites (Fig. 11a). A l'extrémité de certaines d'entre elles, des microfissures de taille d'environ $20 \mu \mathrm{m}$ sont orientées à environ $30^{\circ}$ par rapport à la direction du joint de grain. Elles proviennent vraisemblablement de la pénétration de bandes de déformation dans les joints. C'est probablement à partir de ces microfissures que se développe la fissure de délaminage, le long du joint de grain. En ce qui concerne les états

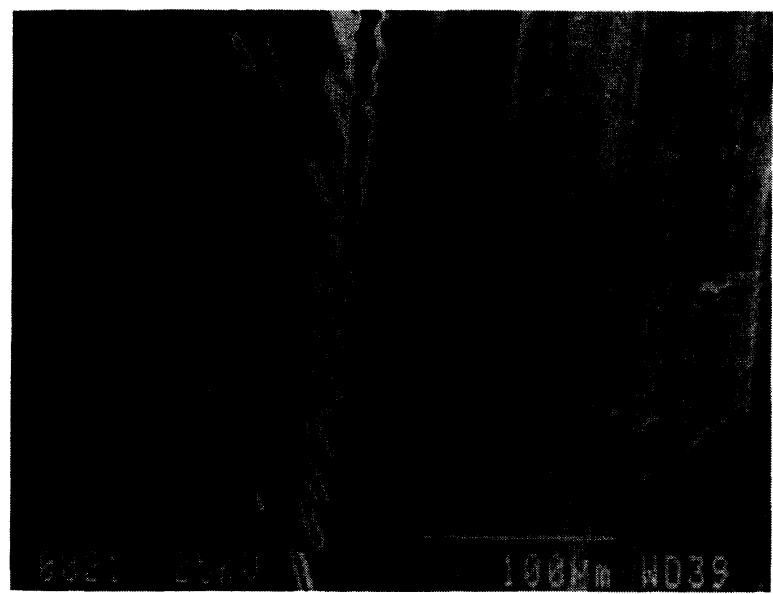

a)

b)

Fig. 11. - MEB, fissures intergranulaires : a) état maturé à température ambiante, b) état sur-revenu.

[SEM micrographs of microcracks at grain boundaries, a) aged at room temperature, b) overaged.] 
fortement revenus, l'allure des fissures de délaminage est différente. En effet, celles-ci sont longues et tortueuses (Fig. 11b), elles cheminent d'un joint de grain à l'autre par l'intermédiaire des points triples. C'est à la rencontre de trois joints de grains où, comme nous l'avons vu précédemment, de gros précipités se forment, que l'amorçage des fissures semble se produire.

\section{Discussion et modélisation de l'amorçage.}

Les relations qui ont été établies entre le glissement et le relief des surfaces de rupture ont permis de mettre en évidence le rôle important que jouent la forte localisation du glissement ainsi que la présence de glissement sinueux particulièrement intense, dans l'amorçage des ruptures intergranulaires. Le glissement sinueux est également à l'origine des ruptures intragranulaires. Nous avons vu, lors de l'étude de la plasticité en surface, qu'au début de la déformation plastique, un seul système de glissement est activé par grain. De ce fait, les incompatibilités de déformation plastique entre grains adjacents, susceptibles de développer un système de contraintes locales complexe, sont sans doute à l'origine de l'activation du glissement sinueux qui va laisser des marches d'une hauteur correspondant au passage d'un grand nombre de dislocations.

Les observations au MET ont aussi montré la forte localisation de la déformation et confirmé l'existence de bandes très intenses qui contiennent un très grand nombre de dislocations accumulées contre les joints de grains. Le fait à noter est que les bandes de déformation observées ne contiennent pas de dislocations. Comme on l'a vu ci-dessus, celles-ci ont dû s'éliminer lors de la préparation de la lame, soit au bord du trou soit dans des fissures intergranulaires. Pour que ces dislocations s'éliminent ainsi, il est nécessaire qu'elles soient dans une configuration qui permette cette élimination, c'est-à-dire qu'elles possèdent peu de liaisons avec d'autres dislocations. Ceci est tout à fait compatible avec une configuration de type empilement de dislocations dans un plan de glissement et contre des joints de grains. Dans ces conditions, on conçoit que la déformation plastique conduit à l'amorçage des fissures aux joints de grains. Cette hypothèse est tout à fait appropriée dans le cas des états sous-revenus, pour expliquer l'amorçage des fissures de délaminage. En effet, des microfissures correspondant à la pénétration de bandes de glissement dans le joint ont été observées à l'extrémité des fissures de délaminage. Dans le cas des états sur-revenus, les traces de glissement, notamment de glissement sinueux sur les surfaces de délaminage, laissent supposer que l'origine de l'amorçage est similaire. Cependant, le nombre de dislocations empilées nécessaire pour amorcer une fissure est évidemment plus faible du fait de la présence d'une grande quantité de précipités inter- granulaires. Ces derniers peuvent favoriser l'amorçage de deux manières différentes: premièrement en augmentant localement le champ de contraintes dû à l'empilement de dislocations et, deuxièmement, en réduisant l'énergie de création de deux surfaces libres nécessaire à la création d'une fissure, en raison de l'existence de l'énergie précipité-matrice.

Les conclusions de cette analyse qualitative suggèrent qu'une approche quantitative de l'amorçage des fissures intergranulaires peut être développée à partir d'un modèle fondé sur les empilements de dislocations aux joints de grains en gardant cependant en mémoire le fait que pour les états revenus au pic et sur-revenus, le champ de contraintes en tête d'empilement peut être partiellement relaxé par déformation plastique dans la zone appauvrie en précipités $\delta^{\prime}$ au voisinage des joints de grains. Aussi le modèle développé ci-dessous n'est en toute rigueur applicable que pour les états maturés et sous-revenus.

Modèle d'amorçage pour les états maturés et sousrevenus :

Considérons un empilement de $N$ dislocations coins contre un joint de grain faisant un angle $\theta$ avec le plan de glissement (voir Fig. 12). Comme cela est fait classiquement, nous considérons des dislocations rectilignes de longueur infinie. Cette hypothèse est bien adaptée au cas du glissement rectiligne; nous supposons qu'il en est de même pour le glissement sinueux bien que cela soit plus discutable. En considérant que le milieu entourant l'empilement est élastiquement isotrope (ce qui apparait justifié dans le cas de cristaux à faible anisotropie comme l'aluminium), l'approche de Stroh [17], permet alors de calculer les contraintes normale $\sigma_{\mathrm{n}}$ et tangentielle $\tau$ dues à l'empilement, en un point $\mathrm{P}$ du joint (Fig. 12). (Les vecteurs normal et

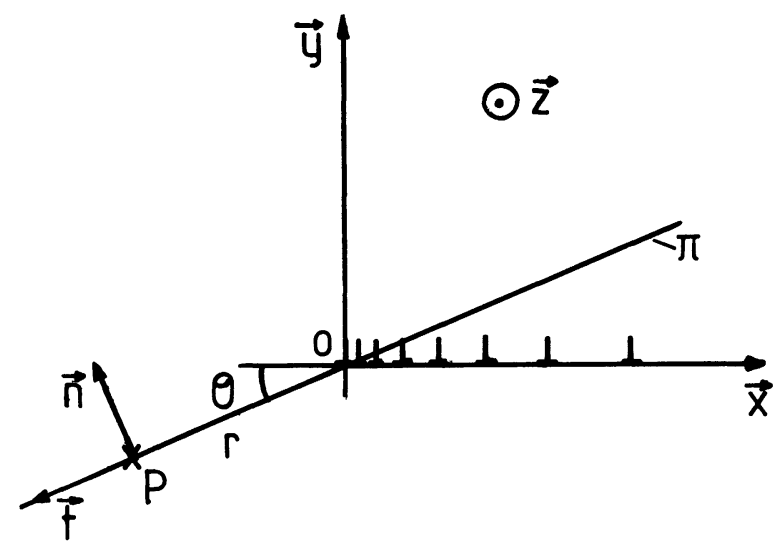

Fig. 12. - Axes de coordonnées définis dans le cas d'un empilement de dislocations contre un joint de grain. L'axe $z$, perpendiculaire au plan de la figure, est contenu dans le plan du joint de grain $\pi$.

[Coordinate axis for dislocations pile up against grain boundary. The $z$ axis, perpendicular to the figure plane, is in the grain boundary plane $\pi$.] 
tangentiel aux joints, respectivement $n$ et $t$, sont définis sur la Fig. 12).

$$
\begin{aligned}
& \sigma_{\mathrm{n}}=\frac{3}{2}\left(\frac{L}{r}\right)^{1 / 2} \tau_{0} \sin \theta \cos \frac{\theta}{2} \\
& \tau=-\left(\frac{L}{r}\right)^{1 / 2} \tau_{0} \cos \frac{\theta}{2}\left(1-3 \sin ^{2} \frac{\theta}{2}\right)
\end{aligned}
$$

avec $L$ la longueur de l'empilement, $r$ la distance du point $\mathbf{P}$ à la dislocation en tête d'empilement, $\tau_{0}$ la scission appliquée sur la source de dislocation.

Sur la figure 13, sont représentées les variations des rapports $\sigma_{\mathrm{n}} / \tau_{0}$ et $\tau / \tau_{0}$ en fonction de l'angle $\theta$ pour une distance $r$ choisie arbitrairement égale à $1 \mu \mathrm{m}$ et pour des grains équiaxes ou de forme allongée.

Dans ce dernier cas, lorsque $\theta$ varie, la longueur d'empilement n'est pas constante mais dépend de l'angle $\theta$. Aussi, nous avons représenté un grain par un parallélépipède de longueur $\ell$ et d'épaisseur $e$, ce qui nous a permis d'obtenir une relation simple entre $L$ et $\theta$.

Dans le cas de grains équiaxes, nous retrouvons le résultat classique où la contrainte maximale est atteinte par la contrainte normale pour un angle $\theta=70,5^{\circ}$. En ce qui concerne les grains allongés et pour les petits angles $\theta$, la contrainte tangentielle $\tau$ devient très grande face à la contrainte normale (Fig. 13).

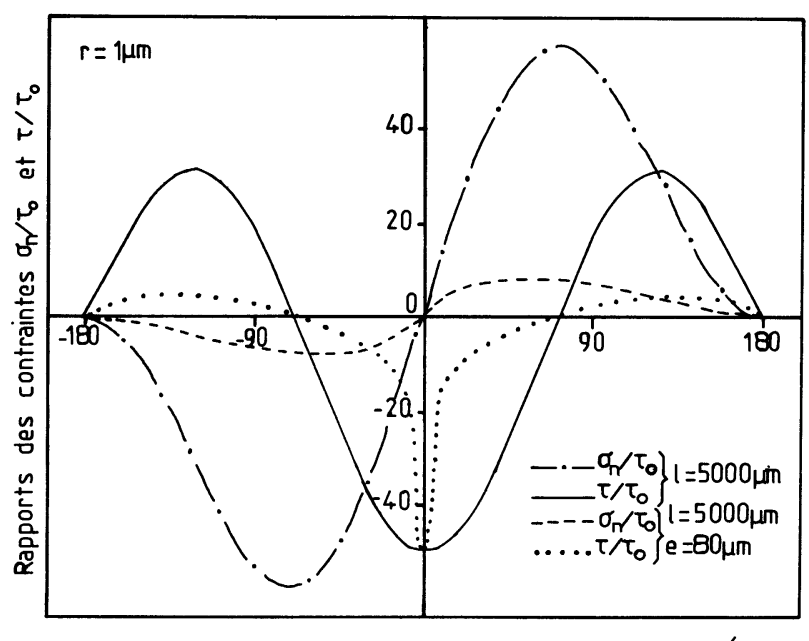

Angle $\theta$ en degrés

Fig. 13. - Contraintes normale et de cisaillement créées

joint de grain (voir Fig. 12), en fonction de l'angle $\theta$ que fait l'empilement avec le joint de grain.

[Normal and shear stresses due to pile up at a given pont $P$ (see Fig. 12) in grain boundary as a function of the angle $\theta$.]

Au vu de ces résultats et du fait que les bandes de glissement peuvent faire un angle assez faible avec les joints de grains, il apparaît nécessaire de développer un modèle d'amorçage tenant compte à la fois de la contrainte normale et de la contrainte tangentielle.

A partir du modèle de Stroh [17], Smith et Barnby
[18] ont déterminé les conditions d'amorçage en modes I et II, soit :

$$
K_{\mathrm{I}}^{2}+K_{\mathrm{II}}^{2}=\frac{\pi^{2} \gamma \mu}{1-\nu}
$$

avec :

- $\gamma$ : l'énergie nécessaire pour créer deux surfaces libres égales à l'unité,

- $\mu$ : le module de cisaillement,

- $\nu$ : le coefficient de Poisson.

$K_{\text {I }}$ et $K_{\text {II }}$ sont respectivement les facteurs d'intensité de contrainte en modes I et II avec, dans le cas d'une fissure de longueur $2 a$,

$$
K_{\mathrm{I}}=\sigma \sqrt{\pi a} \text { et } K_{\mathrm{II}}=\tau \sqrt{\pi a} .
$$

Si on considère que les contraintes $\sigma$ et $\tau$ sont les contraintes $\sigma_{\mathrm{n}}$ et $\tau$ calculées en (1) et que $r=2 a$, il est alors possible de déterminer une scission critique sur la source de dislocation, $\tau_{0 c}$, et un nombre critique de dislocations $N_{\mathrm{c}}$, pour lesquels s'amorce une fissure, soit :

$$
\begin{aligned}
& \tau_{\mathrm{c}}=\left(\frac{\pi \alpha^{\prime} b}{1-\nu}\right)^{1 / 2} \mu L^{-1 / 2} F(\theta)^{-1 / 2} \\
& N_{\mathrm{c}}=\left(\frac{\alpha^{\prime}(1-\nu) \pi^{3}}{b}\right)^{1 / 2} L^{1 / 2} F(\theta)^{-1 / 2}
\end{aligned}
$$

où

$$
\begin{aligned}
F(\theta)=\left(3 / 2 \sin \theta \cos \frac{\theta}{2}\right)^{2}+ \\
\quad\left(-\cos \frac{\theta}{2}\left(1-3 \sin ^{2} \frac{\theta}{2}\right)\right)^{2} .
\end{aligned}
$$

Remarquons que, dans ces relations, la taille des fissures à l'amorçage s'élimine. D'une manière générale, le paramètre $\alpha^{\prime}$ est fonction de l'énergie $\gamma$ définie ci-dessus. L'énergie de surface $\gamma$ requise pour la formation de deux surfaces libres dépend de nombreux paramètres : énergie des joints de grain, présence de précipités, etc. Pour simplifier, nous allons considérer que le joint de grain ne présente pas de précipités. Ce cas correspond à l'état trempé et mâturé à l'ambiante. Kawabata et al. [19] ont défini le paramètre $\alpha^{\prime}$ de la manière suivante :

$$
{ }^{\prime}=\begin{aligned}
1 \gamma_{\mathrm{s}} & \gamma_{\mathrm{j}} \\
2 b \mu & \gamma_{\mathrm{s}}
\end{aligned}
$$

avec :

- $\gamma_{s}$, l'énergie pour créer deux surfaces unité du monocristal de l'alliage considéré,

- $\gamma_{\mathrm{j}}$, l'énergíe du joint de grain. D'après Swalin [20], un ordre de grandeur de $\gamma_{\mathrm{j}}$ peut-être obtenu dans les métaux cubiques faces centrées en considérant que $\gamma_{\mathrm{j}} / \gamma_{\mathrm{s}} \approx 0,35$,

- $b$, le vecteur de Burgers.

En prenant $b=0,286 \mathrm{~nm}, \gamma_{\mathrm{s}}=0,45 \mathrm{~J} / \mathrm{m}^{2}$ [17], $\mu=2,6 \cdot 10^{10} \mathrm{~Pa}, \nu=0,33$, les valeurs numériques 
de $\tau_{0 c}$ et $\mathrm{N}_{\mathrm{c}}$ ont été calculées en fonction de l'angle $\theta$ que fait l'empilement avec le joint, et reportées sur la figure 14. Nous pouvons faire les remarques suivantes :

- pour les angles $\theta$ supérieurs à $40^{\circ}$, environ 400 dislocations sont suffisantes pour amorcer une fissure dans le joint de grain. Ce nombre de dislocations correspond à des hauteurs de marche compatibles avec celles observées au MEB ou avec les cisaillements des joints mis en évidence au MET ;

- pour les angles $\theta$ inférieurs à $40^{\circ}$, le nombre critique de dislocations est plus important, 400 à 1000 , et est toujours comparable aux valeurs déduites expérimentalement des hauteurs de marche de glissement. On peut aussi noter les très faibles valeurs de la scission critique $\tau_{0 c}$.

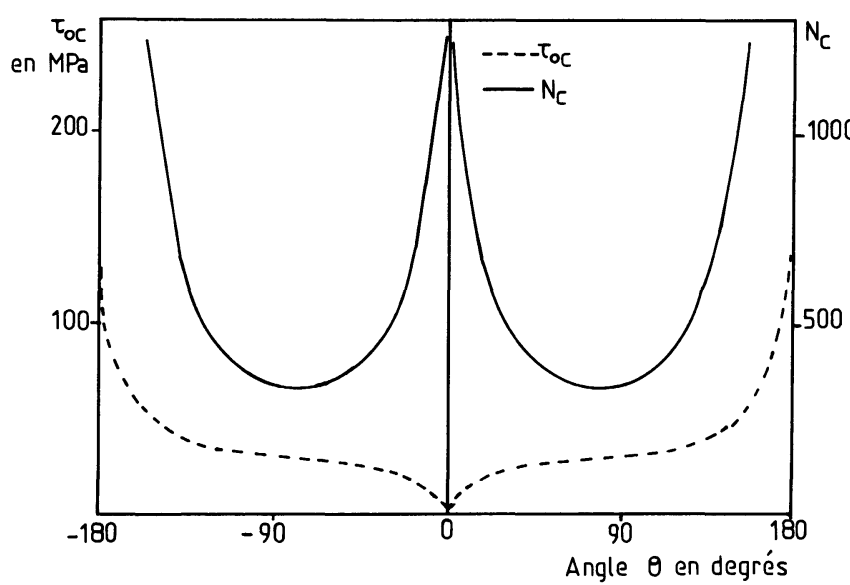

Fig. 14. - Nombre critique de dislocations et scission résolue critique correspondante, provoquant l'amorçage d'une fissure intergranulaire, en fonction de l'angle $\theta$. Cas d'un grain de longueur $\ell=5000 \mu \mathrm{m}$ et d'épaisseur $e=80 \mu \mathrm{m}$.

[Critical number of dislocations and corresponding critical resolved shear stress leading to crack initiation at grain boundaries as a function of the angle $\theta$.]

Ainsi le calcul simplifié effectué dans cet article montre que la forte localisation de la déformation dans des bandes de glissement rectilignes ou sinueuses est à l'origine du délaminage dans les alliages $\mathrm{Al}$ Li. Afin de rendre mieux compte de l'ensemble des mécanismes expérimentaux, il serait nécessaire d'introduire dans le modèle l'effet d'une possible relaxation plastique dans la zone appauvrie en précipités $\delta^{\prime}$ près des joints de grains, et de la présence de la précipitation intergranulaire. Cette dernière peut intervenir de différentes manières:

- tout d'abord, par l'énergie d'interface précipitématrice, $\gamma_{\mathrm{pm}}$, qu'il faut introduire dans l'énergie de surface du joint ;

- ensuite, par le fait que la rupture du précipité peut intervenir avant celle du joint;

- enfin, par la création d'une surcontrainte locale, résultant des précipités, qui peut s'ajouter aux contraintes dues à l'empilement de dislocations.

\section{Conclusion.}

Dans le cas des alliages Al- $\mathrm{Li}$ 8090, nous avons montré que le mode de déformation de ces alliages joue un rôle essentiel lors de l'amorçage des fissures. Les observations au MET ont montré des bandes de déformation très intenses sur un seul système de glissement. Elles laissent des marches sur les joints correspondant au passage de 1000 à 2000 dislocations environ. Du fait probablement des incompatibilités de déformation entre grains adjacents, des glissements sinueux et très intenses sont ensuite activés. Les deux types de glissement sont responsables de l'amorçage des fissures intergranulaires. De plus, les fissures intragranulaires se développent le long des glissements sinueux. La précipitation intergranulaire augmente le risque d'amorçage, mais l'interaction des bandes de déformation avec les joints de grains apparaît tout de même comme étant à l'origine de l'amorçage des fissures. L'analyse quantitative, pour les états maturés ou sous revenus, à partir d'un modèle reposant sur les empilements de dislocation est en bon accord avec les résultats expérimentaux.

\section{Remerciements.}

Nous tenons à remercier le Centre de Recherche de Péchiney-Voreppe qui a financé cette étude, ainsi que le professeur C. Esnouf pour son aide en microscopie électronique à transmission.

\section{Bibliographie}

[1] Wert J. A. et Lumsden J. B., Scr. Met. 19 (1985) 205-209.

[2] Ninomi M., Degawa K. et Kobayashi J., Al-Li IV, Paris (sept. 1987) p. C3 653-659.

[3] Damerval C., Raviart J. L. et Lapasset G., AlLi IV, Paris (sept. 1987) p. C3 661-667.

[4] JATA K. V. et Starke E. A. Jr., Scr. Met. 22 (1988) 1553-1556.
[5] Venkateswara RaO K. T., Hayashi gatani H. F., YU W. et Ritchie R. O., Scr. Met. 22 (1988) 93-98.

[6] Sanders T. H. et Starke E. A., Acta Metall. 30 (1982) 927-939.

[7] Lim F. S., Chakrabortty S. B., Starke E. A., Met. Trans. 13A (1982) 401-410. 
[8] Vasudevan A. K., Miller A. C. et Kersky M. M., Al-Li II (1983) p. 181.

[9] Miller W. S., White J. et Lloyd D. J., Al-Li IV, Paris (sept. 1987) p. C3-139-149.

[10] Meyer P., Cans Y., Ferton D. et Reboul M., AlLi IV, Paris (sept. 1987) p. C3 131-138.

[11] Vasudevan A. K. et Doherty R. D., Acta Metal. 35 (1987) 1193-1219.

[12] Skilingberg M. H. et Ashton R. F., Al-Li IV, Paris (sept. 1987) p. C3 179-186.

[13] Broussaud F. et Diot C., Al-Li IV, Paris (sept. 1987) p. C3 597-603.
[14] Sainfort P. et Dubost B., Al-Li IV, Paris (sept. 1987) p. C3 407-413.

[15] Zhou Z. Q., He X., Ji D. X. et Chen C. Q., Al-Li V, Williamsburg (march 1989) p. 879-888.

[16] Welpmann K., Buhl H., Braun R. et Peters M., Al-Li IV, Paris (sept. 1987) p. C3 677-683.

[17] Stroh A. N., Proc. Roy. Soc. A 223 (1954) 404.

[18] SMith et BARnBY, Met. Sci. J. 1 (1967) 56-64.

[19] Kawabato et al., J. Mat. Sci. 14 (1979) 1071-1079.

[20] Swalin, Thermodynamics of solids, 2nd Fdn (Wiley, New York) 1972, p. 247. 